Jenny Kipper

Pädagogische Elemente in agilen

Methoden - inwieweit lernen

Organisationen im agilen Arbeiten?

\section{Journal für Psychologie}

29. Jahrgang · 2021 · Hett 1

Agile Organisationen

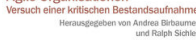

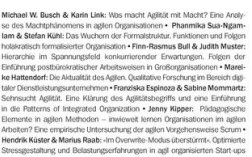

Journal für Psychologie

29. Jahrgang, Nr. 1, 2021, Seite 147-168

Psychosozial-Verlag

DOI: 10.30820/0942-2285-2021-1-147 


\section{Impressum}

Journal für Psychologie

Theorie - Forschung - Praxis

Zeitschrift der Neuen Gesellschaft

für Psychologie (NGfP)

www.journal-fuer-psychologie.de

ISSN (Online-Ausgabe): 2198-6959

ISSN (Print-Ausgabe): 0942-2285

29. Jahrgang, 2021, Heft 1

Herausgegeben von Andrea Birbaumer und Ralph Sichler

https://doi.org/10.30820/0942-2285-2021-1

ISBN der Print-Ausgabe: 978-3-8379-8354-8

ViSdP

Die HerausgeberInnen; bei namentlich

gekennzeichneten Beiträgen die AutorInnen.

Namentlich gekennzeichnete Beiträge stellen nicht in jedem Fall eine Meinungsäußerung der

HerausgeberInnen, der Redaktion oder des Verlages dar.

\section{Herausgebende}

Mag. Andrea Birbaumer, Wien · Dr. Martin Dege, Berlin · Dr. Peter Mattes, Berlin/Wien · Prof. Dr.

Günter Mey, Magdeburg-Stendal/Berlin · Dr. Aglaja

Przyborski, Wien • Paul Sebastian Ruppel, Bochum .

Univ.-Doz. Dr. Ralph Sichler, Wiener Neustadt · Dr.

Anna Sieben, Bochum/Jülich · Prof. Dr. Thomas

Slunecko, Wien

\section{Wissenschaftlicher Beirat}

Prof. Dr. Molly Andrews · Prof. Dr. Thea Bauriedl . Prof. Dr. Jarg Bergold · Prof. Dr. Klaus-Jürgen Bruder • Prof.

Dr. Stefan Busse · Prof. Dr. Tanja Eiselen • Prof. Dr. Jörg Frommer · Prof. Dr. Heiner Keupp · Prof. Dr. Carlos Kölbl · Prof. Dr. Helmut E. Lück · PD Dr. Günter Rexilius · Prof. Dr. Dr. h.c. Wolff-Michael Roth • Prof. Dr. Christina Schachtner - Prof. Dr. Rudolf Schmitt . Prof. Dr. Ernst Schraube • Prof. Dr. Margrit Schreier . Prof. Dr. Hans-Jürgen Seel · Dr. Michael Sonntag . Prof. Dr. Hank Stam · Dr. Irene Strasser, Klagenfurt · Prof. Dr. Dr. Wolfgang Tress • Prof. Dr. Jaan Valsiner - Dr. Barbara Zielke · Prof. Dr. Dr. Günter Zurhorst

\section{Erscheinen}

Halbjährlich als Organ der Neuen Gesellschaft für Psychologie (NGfP) als Open-Access-Publikation und parallel als Print-Ausgabe.

\author{
Verlag \\ Psychosozial-Verlag \\ Walltorstraße 10 \\ D-35390 Gießen \\ info@psychosozial-verlag.de \\ www.psychosozial-verlag.de
}

\section{Abonnentenbetreuung}

aboservice@psychosozial-verlag.de

\section{Bezug}

Jahresabonnement 49,90€ (zzgl. Versand)

Einzelheft 29,90€ (zzgl. Versand)

Studierende erhalten gegen Nachweis 25\% Rabatt, Mitglieder der NGfP erhalten 30\% Rabatt auf den Preis des Jahresabonnements.

Das Abonnement verlängert sich um jeweils ein Jahr, sofern nicht eine Abbestellung bis acht Wochen vor Beendigung des Bezugszeitraums erfolgt.

\section{Anzeigen}

Anfragen richten Sie bitte an den Verlag:

anzeigen@psychosozial-verlag.de

DieZeitschrift Journal für Psychologie wird regelmäßig in der Publikationsdatenbank PSYNDEX des Leibniz-Institut für Psychologie/Leibniz Institute for Psychology (ZPID) erfasst.

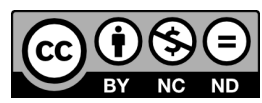

Die Beiträge dieser Zeitschrift sind unter der Attribution-NonCommercial-NoDerivatives 4.0 International Lizenz (CC BY-NC-ND 4.0) lizensiert. Diese Lizenz erlaubt die private Nutzung und unveränderte Weitergabe, verbietet jedoch die Bearbeitung und kommerzielle Nutzung. Weitere Informationen finden Sie unter: creativecommons.org/ licenses/by-nc-nd/4.0/ 


\title{
Pädagogische Elemente in agilen Methoden - inwieweit lernen Organisationen im agilen Arbeiten?
}

\section{Eine empirische Untersuchung der agilen Vorgehensweise Scrum}

\author{
Jenny Kipper \\ Journal für Psychologie, 29(1), 147-168 \\ https://doi.org/10.30820/0942-2285-2021-1-147 \\ CC BY-NC-ND 4.0 \\ www.journal-fuer-psychologie.de
}

\section{Zusammenfassung}

Aus erziehungswissenschaftlicher Sicht analysiert dieser Beitrag, welche Lernformen und pädagogischen Technologien im Alltag von Scrum-AnwenderInnen auffindbar sind, sodass von einer lernenden Organisation gesprochen werden könnte. Nach einer theoretischen Analyse des Scrum Guide folgen Einblicke in acht ExpertInneninterviews, die mittels einer rekonstruktiven Analyse auf vorhandene pädagogische Ansätze, Technologien und Lernindikatoren untersucht werden, bevor abschließend der Hypothese > wenn eine Organisation agil arbeitet, lerne sie auch< nachgegangen wird.

Schlüsselwörter: Agile, Scrum, Organisationslernen, Lernen, Organisationen

\section{Summary}

Pedagogical elements in agile methods - How far learn organisations within agile working?

An empirical analysis of the agile framework Scrum

From the perspective of educational sciences this contribution will focus on forms of learning and pedagogical technologies that are visible in the daily routine of scrum users. The theoretical analysis of the scrum guide is followed by insights into eight interviews with experts. These have been analysed to find pedagogical aspects, technologies and indicators for learning. Finally, there will be a discussion on the statement that each agile working organisation is at the same time a learning organisation.

Keywords: Agile, Scrum, organisational learning, learning, organisation 


\section{Agilität und Erziehungswissenschaften - Möglichkeiten der Verknüpfung?}

Wenn man heutzutage über Organisationen liest, dann meist im Zusammenhang mit dem Phänomen Agilität - und das schon lange nicht mehr nur in Bezug auf IT-Unternehmen (Komus et al. 2020, 182). Agilität scheint in der Arbeitswelt 4.0 ein fast unverzichtbarer Bestandteil. Inkludiert ist das Bewusstsein, dass die Bedeutung der Technologie genauso wichtig ist wie die Kommunikation von Mensch zu Maschine und besonders auch von Mensch zu Mensch (vgl. Negri 2019, 5). Letzteres betont zum Beispiel auch das »Agile Manifest « mit seinen dahinter liegenden Prinzipien, das seit 2001 ein zentrales Arbeitsdokument für viele Organisationen, mittlerweile auch außerhalb der Softwareentwicklung, ist (Beck et al. 2001). Besonders im Jahr 2020, das durch die Covid-19-Pandemie geprägt war, ging es im organisationalen Kontext meist darum, agil, flexibel, hierarchielos oder selbstorganisiert zu werden oder zu sein. Angelehnt an Pütz und Ricker $(2017,17)$ ist Agilität verkürzt gesprochen »die Transformationsfähigkeit des Unternehmens, also die Möglichkeit oder Fähigkeit, sich schnell und wirkungsvoll zu verändern «. Erste Definitionen im sozialwissenschaftlichen Kontext finden sich bereits seit den 1950er Jahren und stützen sich auf die Theorie selbstorganisierter Systeme (Förster und Wendler 2012). Wege in die Agilität gibt es viele und genauso viele BeraterInnen. Die gängigen Management- und Organisationsentwicklungszeitschriften vermitteln den Eindruck, dass mittlerweile fast jedes Unternehmen agil arbeitet oder es zumindest versucht.

Aus einer erziehungswissenschaftlichen Sicht steckt hinter einer Veränderung Lernen (vgl. Siebert 2010, 190f.). Damit eine Organisation sich verändern, zum Beispiel agil werden kann, müssen die Individuen in ihr lernen (Kipper 2014). Insofern lohnt die erziehungswissenschaftliche Betrachtungsweise agiler Phänomene, die versprechen, dass eine agile Organisation eine lernende Organisation ist (Pfister und Müller 2019, 49). Dieses Lernen in Organisationen wird realisiert durch pädagogische Technologien (»Mittel mit der Intention des pädagogischen Handelns «; Kipper 2014, 159), die sich in den Subkategorien Programme, Arbeits- und Veranstaltungsformen, Methoden und Medien wiederfinden (vgl. Kipper 2014, 159ff.; Nittel et al. 2020). (Im vorliegenden Artikel werden die Begriffe »Organisation « und »Unternehmen « synonym verwendet.)

Wenn also die Rede von einer Organisationsveränderung ist, lohnt es sich nachzuvollziehen, wie und wo das Lernen hin zu der neuen Verhaltensweise erfolgt und welche Lernformen und pädagogische Technologien im Alltag der AnwenderInnen zum Tragen kommen. Dieser Frage wird im vorliegenden Artikel nachgegangen. Dabei steht nicht die Einführung von Scrum im Vordergrund, sondern das tägliche Arbeiten damit. Insofern wird hinterfragt, inwieweit der tatsächliche Arbeitsalltag mit Scrum eine erziehungswissenschaftliche Verbindung aufweist. 
Innerhalb der Ansätze, die ein agiles Arbeiten im organisationalen Kontext fördern, weist Scrum »die größte allgemeine Bedeutung auf. 55\% der agilen Teilnehmer bewerten den Ansatz als sehr wichtig und 29\% als wichtig für ihren Bereich, nur ungefähr $14 \%$ hingegen als unwichtig oder von geringer Bedeutung « (Komus et al. 2020, 52). Dies konstatieren Komus et al. in ihrer aktuellen Studie mit einer Stichprobengröße von $n=642$ und ergänzen: Mit $\gg 84 \%$ ist Scrum weiterhin der meistgenutzte agile Ansatz auf Teamebene « $(2020,3)$.

Diesen Eindruck kann man auch bei der Betrachtung der Literatur zu agilen Herangehensweisen gewinnen. Deshalb wurde Scrum als eines der populärsten agilen Phänomene zu einer ersten Analyse in diesem Stil von der Autorin herangezogen. Dafür erfolgt zuerst eine lerntheoretische Betrachtung, die durch eine empirische Analyse ergänzt wird. Acht ExpertInneninterviews dienen als Forschungsmaterial, um sich der agilen Arbeitsweise Scrum anzunähern und zu rekonstruieren, auf welchen Ebenen das organisationale Lernen stattgefunden hat, bzw. hätte stattfinden können.

Damit verfolgt die Autorin das Ziel, die Diskussion rund um agile Vorgehensweisen auf der Basis von empirischen Ergebnissen zu führen, auch wenn klar ist, dass eine wesentlich größere Datenmenge nötig wäre, um repräsentative Aussagen treffen zu können. An dieser Stelle soll so erst einmal ein Einstieg in den Diskurs gelingen.

Bei der Sichtung der bisherigen deutschsprachigen Veröffentlichungen ist auffällig, dass über agiles Arbeiten im organisationalen Kontext und besonders über Scrum überwiegend Ratgeber- oder Managementliteratur vorhanden ist, Scrum jedoch bis jetzt keiner fundierten sozialwissenschaftlich-empirischen Analyse unterzogen wurde.

Auffindbar sind Publikationen überwiegend aus Psychologie (z. B. Pfister und Müller 2019), Betriebswirtschaft (z. B. Peters et al. 2019, 2020) sowie anwendungsbezogene Fach- und Ratgeberliteratur (z.B. Maximini 2018). Die vorhandenen Studien geben meist einen Überblick über die Marktdurchdringung agiler Herangehensweisen (z. B. Komus et al. 2020) oder auch Erfolgsfaktoren (z. B. Böhm 2019) und Zufriedenheit (z.B. Komus 2013), beschäftigen sich dagegen eher weniger mit qualitativer Belegbarkeit von Wirksamkeit oder Auswirkungen. Auch kann konstatiert werden, dass vorhandene Studien meist in Kooperationen entstanden sind, zum Beispiel von Forschungsinstituten und Beratungsunternehmen (z. B. Peters et al. 2019, 2020; Pütz und Ricker 2017; Rumpp et al. 2018). Ebenfalls vorhanden sind Praxisbeispiele und Fallgeschichten (z. B. Koschek 2014, 2018). Laut Komus et al. werden im Markt vor allem »Fragen, wie Nachhaltigkeit in der Nutzung, umfassende Nutzung in der gesamten Organisation, erfolgreiche Ausgestaltung von Mischformen ( $>$ hybrid<, > selektiv<) und vor allem die Ausgestaltung agiler Systeme über das einzelne Team hinaus (>agile Skalierung<) « diskutiert (2020, 8). Ebenso steht es um die Implementierung agiler Arbeitsformen und deren Erfolgsfaktoren. Dies findet sich auch in der englischsprachigen Literatur, wo zum Beispiel bei Krancher, Luther und Jost (2018) aus IT-Perspektive lern- 
theoretische Aspekte gestreift werden oder bei Vidgen und Wang (2009) agiles Arbeiten mittels komplexitätstheoretischer Überlegungen beleuchtet wird. Jedoch stellen all diese Schriften das Lernen und seine tatsächlichen Ausprägungen (Formen, Bedingungen, Technologien) nicht kleinschrittig in den Mittelpunkt und machen so die Lernräume für Individuen und Organisation nicht nachvollziehbar. (Die Autorin erhebt an dieser Stelle keinen Anspruch auf Vollständigkeit des dargestellten Forschungsstandes. Vielmehr ist dies eine rudimentäre Darstellung des Marktes, da der Fokus auf dem erziehungswissenschaftlichen Interesse liegt.)

Die Erziehungswissenschaft, die aus lerntheoretischer Sicht ein großes Interesse an dem Diskurs zeigen müsste, ist bis jetzt noch nicht sichtbar involviert. Lernen ist ein pädagogischer Schlüsselbegriff (zum Diskus siehe Dinkelaker 2018, 50ff.), denn Erziehung und Bildung wären ohne Lernen nicht möglich (vgl. Koch 1999, 351ff.). Da die Diskussion über Lernen mittlerweile unter dem Stichwort Lebenslanges Lernen (vgl. Nittel 1996, 738) auch Lernen in nicht explizit pädagogischen Kontexten umfasst (Kipper 2014), ist es unumgänglich, dass sich vor dem Hintergrund von zum Beispiel selbstorganisiertem Lernen, Lernen im Alltag oder autodidaktischem Lernen (vgl. Kade et al. 1999, 31) der erziehungswissenschaftliche Diskurs auf den Grundlagen der Organisationspädagogik (Geißler 2000) auch den neueren Phänomenen rund um Agilität widmet, vor allem da anscheinend den MitarbeiterInnen innerhalb der sich ständig verändernden Organisationen bewusst zu sein scheint, dass sie fortlaufend neue Kompetenzen erwerben müssen, die nicht zwingend immer über klassische Weiterbildungen erlernbar sind (vgl. Peters et al. 2020, 30f.). 2019 war ein weiteres Ergebnis der jährlichen Studie von Peters et al., dass Organisationen zwar agil arbeiten, Agilität jedoch noch $\gg$ nicht in den Köpfen ihrer Mitarbeitenden und in ihrer Unternehmenskultur verankert « ist (2019, 4). Beides, Lernen von Individuen und Lernen von Organisationen zur Verankerung solcher Veränderungen, sind originär erziehungswissenschaftliche Aufgabengebiete.

\section{Scrum aus erziehungswissenschaftlicher Sicht - Eine theoretische Analyse}

»Um sich den Gegebenheiten des Lebens und der Umwelt anzupassen, ist die Fähigkeit zu Lernen eine Grundvoraussetzung. Lernen zu können ermöglicht einem, sinnvoll und zielgerichtet in einer sich verändernden Umwelt zu agieren «, leiten Pfister und Müller ihren Beitrag über Lernpsychologie und Agilität ein (2019, 34). Darin analysieren sie agile Arbeitsprinzipien und erläutern die enthaltenen Grundzüge aus Konditionierung, Lernen am Modell und Lernen durch Einsicht (ebd. 2019). Dies gilt sowohl auf einer individuellen als auch auf einer organisationalen Lernebene, so zum Beispiel durch Modelllernen, resümieren Pfister und Müller, welches dadurch schon belegt sei, dass agiles 
Arbeiten aus der Softwarebranche via best practise sharing auf Unternehmen anderer Branchen übertragen wurde (vgl. ebd., 34).

Es lohnt sich, das Thema genauer zu betrachten. Die Grundidee der Handlungspraxis ist, dass eine Organisation, die agil arbeitet, gleichzeitig auch lernt. Agilität ist auf der organisationalen Ebene die Anpassungsfähigkeit an sich wandelnde Umweltbedingungen (vgl. ebd., 39). Agiles Arbeiten ist dabei als die Verankerung auf individueller Ebene zu sehen. Nach Pfister und Müller sind agile Vorgehensweisen so aufgebaut, dass sie grundlegende psychologische Lernprozesse berücksichtigen und dadurch individuelle und kollektive Verhaltensveränderungen ermöglichen (vgl. ebd., 49f.).

Scrum ist ein Weg, agil zu arbeiten. Die Entwickler von Scrum, Ken Schwaber und Jeff Sutherland, bezeichnen Scrum als $\gg$ Rahmenwerk zur Entwicklung, Auslieferung und Erhaltung komplexer Produkte «, das Menschen »in die Lage versetzt [...], produktiv und kreativ Produkte mit höchstmöglichem Wert auszuliefern « und dabei » fortlaufend das Produkt, das Team und die Arbeitsumgebung [zu] verbessern « (2017, 3). Insofern könnte man also vermuten, dass die Grundidee von einer lerntheoretischen Idee und Lernphasen (Giesecke 2015) nicht weit entfernt sei, da für konstante Verbesserungen Lernen in unterschiedlichen Ausprägungen und Phasen notwendig sein sollte. Auch beim Lernen ist die Ungewissheit über Prozess und Ausgang präsent, ähnlich zu Scrum.

Das Vorgehen nach Scrum ist gekennzeichnet durch Iterativität, hohe Kommunikation im Team sowie zeitliche und finanzielle Rahmung (Duméril 2019). Die Erfinder Schwaber und Sutherland bezeichnen das Vorgehen als Regelwerk, das »die Beziehungen und Wechselwirkungen zwischen den Rollen, Ereignissen und Artefakten bestimmt « $(2017,3)$ und auf den drei Säulen empirischer Prozesssteuerung (Transparenz, Überprüfung und Anpassung) basiert (vgl. ebd., 4).

\subsection{Allgemeine lerntheoretische Verknüpfungsmöglichkeiten}

Wie bereits vorab erläutert, ist Lernen als erziehungswissenschaftlicher Kernbegriff die Grundlage für Veränderung. Die Arbeitsweise Scrum ermöglicht Organisationen, agil zu arbeiten. Im Vorgehen nach Scrum können lerntheoretische Konzepte, pädagogische Kernaktivitäten und Technologien sowie lernbezogene Rollen auf einer mikrodidaktischen Ebene erkannt werden. Die nachfolgende Darstellung fokussiert lediglich auf Basis der Scrum-Theorie die Ereignisse und Rollen, und bietet keine umfassende Erläuterung des Prozesses.

Die Ereignisse:

> Sprint: Der Sprint ist »ein Projekt mit einem Zeithorizont von maximal einem Monat « (Schwaber und Sutherland 2017, 9), vergleichbar mit einer Lernzielorien- 
tierung (Siebert 2012, 149ff. oder Traub und Konrad 2016, 57ff.). Der Weg zum jeweiligen Ziel ist bei beiden flexibel und beinhaltet Zwischenüberprüfungen bezüglich der Zielerreichung.

> Sprint Planning: Scrum sieht vor, dass innerhalb von einem Tag die Inhalte des anstehenden Sprints in einem Sprint Planning vom Team geplant werden. Der Vergleich mit einer Lernform könnte zu selbstgesteuertem und kooperativem Lernen führen, denn beim selbstgesteuerten Lernen bestimmt der »Lerner [...] das Lernziel, den Lerninhalt, die Lernform und fordert bei Bedarf den Lernberater an « (Deitering 1995, 10). Der Scrum Master fungiert dabei als Lernberater. Ziel des Sprint Planning ist es, dass das Scrum-Team erläutern kann, » wie es als selbstorganisiertes Team an der Erreichung des Sprint-Ziels und der Erstellung des gewünschten Produktinkrements arbeiten möchte « (Schwaber und Sutherland 2017, 11). Fachliche Expertise kann von außerhalb des Teams hinzugezogen werden. Eine Vergleichbarkeit des Teamlernens im Sprint Planning kann allerdings auch mit der Arbeit in heterogenen Lerngruppen im Rahmen von kooperativem Lernen hergestellt werden (»eine Interaktionsform, bei der die beteiligten Personen gemeinsam und in wechselseitigem Austausch Kenntnisse und Fertigkeiten erwerben «; Konrad und Traub 2010, 5). Beides erfordert TeilnehmerInnenorientierung (vgl. Siebert 2012, $117 \mathrm{ff}$.) bzw. das Berücksichtigen individueller Kompetenzen (Traub und Konrad 2016, 53ff.). Letzteres hat das Ziel, als Gruppe ein Resultat gemeinsam, jedoch mit Aufgabenteilung, zu erarbeiten und sich hierfür zu unterstützen sowie voneinander und im sozialen Austausch zu lernen. Beides ist allerdings eher im Bereich des informellen Lernens im Arbeitsalltag (vgl. Dohmen 2001, 47ff.) anzuordnen.

> Sprint-Ziel: Das Sprint-Ziel ist ein Zwischenziel auf dem Weg zum Gesamtprodukt, vergleichbar mit der Orientierung an einem Lernziel.

$>$ Daily Scrum: Das Entwicklungsteam trifft sich täglich, um in einer 15-minütigen Besprechung zu erörtern, wie der vergangene Tag hinsichtlich der Zielerreichung gelaufen ist und wie die nächsten 24 Stunden gestaltet werden sollen. Der Scrum Master organisiert den Rahmen für die Zusammenkunft und die Einhaltung der Scrum-Regeln. Die Art und Weise der Besprechung hängt vom Team ab. Das Setting erinnert ebenfalls an selbstorganisiertes kooperatives Lernen bei dem ein/e MentorIn als BegleiterIn und OrganisatorIn fungiert (zur pädagogischen Kernaktivität des Begleitens und Organisierens siehe Nittel et al. 2020). Die Fragen, die in der Besprechung beantwortet werden sollen (vgl. Schwaber und Sutherland 2017, 12), erinnern ebenfalls stark an eine Lernzielorientierung, wobei hier auch erste Bezüge zum Erfahrungslernen vermutet werden können (vgl. Dinkelaker 2018, 57ff.).

$>$ Sprint Review: Dieses Ereignis dient der Überprüfung der Zielerreichung. Das Ergebnis des Sprints wird begutachtet, der Weg dahin erörtert und nächste Schritte sortiert sowie geplant, damit die Zielerreichung realisierbar bleibt. Damit ist das 
Sprint Review vergleichbar mit einem selbstgesteuerten Selbstbewertungsprozess (vgl. Hörmann 1996, 149ff.) und am Erfahrungslernen Deweys orientiert (vgl. Dinkelaker 2018, $57 \mathrm{ff}$. oder Göhlich 2014a, 191ff.).

$>$ Sprint Retrospektive: Die Elemente der Sprint Retrospektive (vgl. Schwaber und Sutherland 2017, 14) beinhalten für das gesamte Scrum-Team inklusive des Scrum Master sowohl reflexive als auch vorausschauende Diskussionselemente, um eine Optimierung des nächsten Sprints zu ermöglichen. Der Scrum Master fungiert hier auch wieder in einer rahmengebenden Rolle. »Auch wenn jederzeit Verbesserungen eingeführt werden können, bietet die Sprint Retrospektive eine formelle Gelegenheit, sich auf die Überprüfung und Anpassung zu fokussieren « (ebd.). Darüber hinaus ist dieses Ereignis explizit zum Lernen, mit dem Ziel einer Verbesserung, vorgegeben. Durch seinen reflexiven Charakter besteht möglicherweise die Möglichkeit zum selbstorganisierten Erfahrungslernen oder Um- und Dazulernen (vgl. Schwarzer und Buchwald 2014, 213ff.) bis hin zum transformativen Lernen (vgl. Zeuner 2012, 93ff.). Besonders in diesem Schritt sind die enthaltenen Lernprozesse relevant, damit neben dem agilen Arbeiten auch Agilität als Haltung ausgeprägt werden kann.

Die Rollen des Scrum Master:

$>$ Er hat eine eher unterstützende Rolle für den Product Owner und das ganze Scrum-Team.

$>$ Er hilft beim Verständnis der Arbeitsweise Scrum und der Umsetzung in der Teamzusammenarbeit, zum Beispiel in der Selbstorganisation (vgl. Schwaber und Sutherland 2017, 8). Diese Rolle ist ähnlich der Rolle des/der Mentors/Mentorin, Begleiters/Begleiterin oder Organisators/Organisatorin für das Team.

Durch alle Scrum-Ereignisse hinweg sind seine pädagogischen Kernaktivitäten die des Begleitens, Beratens und Organisierens (zu pädagogischen Kernaktivitäten siehe Nittel et al. 2020). Dabei formulieren die Erfinder auch direkt, dass diese Arbeit Lernen und Lernförderung enthält:

$\gg$ Die Aufgabe des Scrum Masters ist es, mit dem Scrum-Team und der Organisation an der Verbesserung der Transparenz der Artefakte zu arbeiten. Diese Arbeit bedeutet zumeist Lernen, Überzeugen und Verändern. Transparenz fällt einem nicht in den Schoß, sie ist ein Weg, den es zu beschreiten gilt« (Schwaber und Sutherland 2017, 17).

Demzufolge kann sogar konstatiert werden, dass hier eine Aufforderung zu einem expliziten pädagogischen Handeln gegeben ist (zu pädagogischem Handeln vgl. Giesecke 2015, 20ff.). 
Auch wenn das eigentliche Ziel bei dieser Form der agilen Arbeitsweise kein ursprünglich pädagogisches ist, so weist die theoretische Vorgehensweise doch eine Nähe zu der Idealstruktur pädagogischen Handelns auf:

$\gg 1$. Festlegung des Zieles;

2. Diagnose der Situation, in die hinein gehandelt werden soll;

3. Antizipation des Ablaufes;

4. Prüfung des Ergebnisses;

5. Beendigung oder Korrektur des Lernprozesses « (Giesecke 2015, 56).

Gegebenenfalls kommt es noch mal zu einer Segmentierung des Zieles in Unterziele. $\mathrm{Zu}$ Beginn des Prozesses stehen Erwartung, Motivation, Lernfähigkeit und Ressourcen der beteiligten Individuen im Vordergrund. Bei Scrum wären dies die Kompetenzen und Ressourcen.

Wie diese kurze Analyse des Scrum Guides (Schwaber und Sutherland 2017) zeigt, kann sowohl sprachlich als auch bezogen auf die Abläufe eine Nähe zum Lernen, zu Lernformen und ihren Bedingungen hergestellt werden. Insofern könnte aufgrund der dadurch gegebenen Bedingungen angenommen werden, dass die Mitglieder eines Scrum-Teams in ihrem Arbeitsalltag viele Möglichkeiten zum Lernen bekommen, vor allem deshalb, weil ihr Handeln durch pädagogisches Handeln des Scrum Masters begleitet wird. Durch seine Tätigkeiten des Begleitens, Beratens und Organisierens eröffnet er Lernräume.

\subsection{Verknüpfungsmöglichkeiten zu pädagogischen Technologien}

Explizit pädagogisch intendierte Technologien sind im Scrum-Regelwerk genauso wenig zu finden wie explizit intendierte Lernformen. Jedoch können auch hier auf den zweiten Blick Bezüge zu impliziten Lernräumen hergestellt werden. Scrum wird in seiner ursächlichen Bezugswissenschaft und -literatur als »Rahmenwerk « beschrieben (Schwaber und Sutherland 2017, 3). Pädagogisch gesehen könnte man es auf der makrodidaktischen Ebene als Programm einstufen: »ein organisatorisch, technisch und räumlich aufwendiges Lernarrangement, in dem die Kombination mehrerer Veranstaltungsformen, Methoden und Medien zu einem bestimmten Zweck stattfinden kann « (Kipper 2014, 161). Veranstaltungsformen benötigen »einen professionellen Verfahrensverwalter für die stattfindenden Vermittlungs- und Aneignungsprozesse « (ebd., 161) und sind damit nach Frey »Rahmung für Lehr- und Lernprozesse «, die »Spielräume für didaktisches Handeln « eröffnen und gleichzeitig »die Fülle an pädagogischen Handlungsoptionen « begrenzen (Frey 2012, 356). Hierunter fallen zum Beispiel das Sprint Review oder die Sprint Retrospektive. Innerhalb dieser Veranstaltungsformen könnten verschiedene Me- 
thoden eingesetzt werden. Methoden sind im pädagogischen Sinne »Vorgehensweisen zur Erreichung von Erziehungs- und Bildungszielen « (Reinhold et al. 1999, 374). Sie sind nicht gegenstandsbezogen, meist regelgeleitet und beschreiben den Weg zu einem Lernziel. Im Rahmen des Methodeneinsatzes besteht wiederum die Möglichkeit, verschiedene Medien einzusetzen. Medien als zweckgebundene und gegenstandsbezogene Mittel stehen in Relation zu den pädagogischen Zielen der jeweiligen Methode (vgl. Friedrichs 2012, 347f.). Im Rahmen der Scrum-Ereignisse könnten sowohl Methoden als auch Medien mit implizitem pädagogischen Bezug genutzt werden. Diese sind jedoch nicht im Scrum Guide (Schwaber und Sutherland 2017) vorgegeben. Eine Betrachtung der pädagogischen Technologien zeigt auf, dass auch hierüber Lernräume angeboten werden.

\subsection{Verknüpfungsmöglichkeiten zum organisationalen Lernen}

Wenn nun die Scrum-Teammitglieder den sich ihnen bietenden Lernraum nutzen und darin jeder für sich und auch alle zusammen lernen würden, dann müsste theoretisch organisationales Lernen entstehen, insofern die daraus resultierenden Veränderungen Konstanz aufweisen und sich auf organisationale Prozesse und Strukturen auswirken, auch wenn das kollektive sowie organisationale Lernen nicht vordergründig avisiert wurde (zu organisationalem Lernen siehe Geißler 2000; Göhlich 2014b; Kipper 2014).

Es könnte zum Beispiel vermutet werden, dass durch den gesamten Scrum-Prozess hindurch Organisationswissen als Resultat individueller und kollektiver Lernprozesse entstünde und die Basis für Organisationslernen bilde (Geißler 1995).

Mit March und Olsen könnte angenommen werden, dass organisationales Lernen aus kollektiven Realitätsdeutungen und -bewertungen (vgl. Geißler 1995, 41ff.) zum Beispiel in Scrum-Ereignissen hervorgehe.

Argyris und Schön (1996) würden vielleicht aufdie Suche danach gehen, ob die ScrumEreignisse single-loop-learning-, double-loop-learning-oder deutero-learning-Prozesse hervorrufen würden (vereinfacht gesprochen Reflexionsschleifen zwischen Handlung und Resultat, oder zwischen Resultat und Handlung und Zielen; oder zwischen Resultat und Handlung und Zielen sowie einer zusätzlichen Reflexion und Analyse des Lernprozesses) und entsprechend Rückkopplungen auf Handlungs-, Ziel- oder Reflexionsebene ergäben. Letztere könnten zum Beispiel aus und in Sprint Retrospektiven entstehen.

\subsection{Fazit der theoretischen Analyse}

Zusammenfassend lässt sich nach der theoretischen Betrachtung des Scrum-Regelwerkes festhalten, dass innerhalb der vorgegebenen Struktur verschiedenste Lernformen 
und pädagogische Kernaktivitäten enthalten sind, jedoch implizit und nur selten explizit. Demzufolge sind individuelle Lernräume vorhanden, die wiederum zu kollektivem Lernen und schließlich einer lernenden Organisation während und nach der Anwendung von Scrum führen könnten. Scrum als Programm beinhaltet verschiedene Veranstaltungsformen, innerhalb derer implizit pädagogisch intendierte Methoden und Medien zur Anwendung kommen können und deren Verwendung somit auch zu individuellem und kollektivem Lernen beitragen könnte.

Insofern ist abschließend nachvollziehbar, wenn davon gesprochen wird, dass eine agil arbeitende Organisation gleichzeitig auch eine lernende Organisation ist - zumindest aus theoretischer Perspektive. Ob ein organisationaler Lernprozess vonstattengeht, müsste durch eine langfristige empirische Überprüfung erst bestätigt werden.

Nachfolgend dargestellt ist ein erster qualitativer Versuch über die Handlungspraxis nachzuvollziehen, inwieweit die vielfältigen Lernräume zum Lernen beitragen. Ziel ist es nicht, und das kann auch auf Basis von acht ExpertInneninterviews nicht repräsentativ gewährleistet werden, eine der oben genannten Theorien im Material nachzuweisen. Ziel ist es vielmehr, das Datenmaterial auf Anhaltspunkte für organisationale Lernprozesse zu untersuchen und somit eine erste explorative Untersuchung des Phänomens vorzulegen.

\section{Scrum aus erziehungswissenschaftlicher Sicht - Eine qualitative Analyse}

\subsection{Methodisches Vorgehen}

Um in die Handlungspraxis von Organisationen und in die der in ihnen agierenden Akteure blicken zu können, wurden acht narrative ExpertInneninterviews (Meuser und Nagel 1994) geführt. Die Interviews mit ExpertInnen aus unterschiedlichen Organisationen bieten einen variantenreichen Blick auf das Phänomen Scrum.

Der Autorin ist bewusst, dass organisationales Lernen eigentlich nur durch Methodentriangulation und das Handeln der einzelnen Organisationsmitglieder nur über Beobachtungen analysiert werden kann. Die dem Artikel zugrunde liegende Forschung löst diese Herausforderung, indem die Beschreibungen der Handlungspraxis in den ExpertInneninterviews stellvertretend gedeutet werden.

Ein offener Leitfaden mit fünf thematischen Fragenblöcken sorgte bei den Interviews für Vergleichbarkeit und gewährleistete gleichzeitig einen hohen narrativen Anteil seitens der Interviewten (Meuser und Nagel 1994). Durch die gestellten Fragen wurden die GesprächspartnerInnen aufgefordert, Beispiele aus ihren Scrum-Erfahrungen zu berichten und zu differenzieren, in welchen Situationen sie das Arbeiten nach Scrum als anders erlebt haben, inwiefern der Wechsel zur agilen Projektmethode einen 
Mehrwert gebracht hat oder auch wann ein Weiterarbeiten nach der »alten Vorgehensweise « hilfreicher gewesen wäre. Des Weiteren enthielt ein Fragenblock unter anderem die Aufforderung, über Veränderungen in Abläufen und das Teamerleben $\mathrm{zu}$ reflektieren. Besonders die Belegbeispiele, das detaillierte Schildern des eigenen Erlebens/Handelns und die Reflexion über Veränderungen lassen so retrospektiv die Analyse der Handlungspraxis und damit des Lernens auf individueller und organisationaler Ebene möglich werden, ohne direkt auf eine Lernform zu fokussieren und Platz für persönliche Relevanzsetzungen zu geben.

Da die Corona-Krise bei den meisten GesprächspartnerInnen kein persönliches Gespräch erlaubte und die ExpertInnen in ganz Deutschland verstreut waren, erfolgte die Interviewdurchführung telefonisch oder via eines Webkonferenz-Tools. Die Interviewten stimmten im Vorfeld des Interviews einer Aufzeichnung und Auswertung des Gespräches schriftlich zu. Kriterien für die Auswahl der InterviewpartnerInnen waren

$>$ Erfahrungen in der Arbeit nach/Anwendung von Scrum oder/und eine Weiterbildung zu Scrum,

$>$ Erfahrungen im klassischen Projektmanagement und

> Projekt-/Produkt- oder Managementperspektive auf Scrum.

Diese Kriterien sind insofern wichtig, als die Schilderungen der Handlungspraxis eine rekonstruktive Analyse hinsichtlich pädagogischer Ansätze, Technologien, Aktivitäten und Lernindikatoren auf individueller und organisationaler Ebene zulassen.

Darüber hinaus wurde auf einen Mix aus männlichen und weiblichen InterviewpartnerInnen mit einer Altersverteilung zwischen Mitte 30 und Mitte/Ende 50 geachtet, die verschiedenen Branchen sowie unterschiedlichen hierarchischen Ebenen zugehörig sind, bzw. die Sichtweise angestellt versus freiberuflich oder auch Scrum-Weiterbildung versus keine Weiterbildung, damit möglichst viele Perspektiven auf den Forschungsgegenstand möglich sind.

Das Sampling ist wie folgt strukturiert (aus Anonymitätsgründen sind nur einige Auswahlfaktoren angeführt, die zum Zeitpunkt des Interviews aktuell waren):

> Interview A: männlich, IT-Projektmanager, freiberuflich, Managementperspektive;

> Interview B: weiblich, IT-Projektmanagerin, angestellt, Großunternehmen Telekommunikationsbranche, Projektperspektive;

> Interview C: männlich, IT-Leiter, Großunternehmen Automobilbranche, angestellt, Managementperspektive;

> Interview D: männlich, Projektmanager, angestellt, Großunternehmen Finanzbranche, Projektperspektive;

> Interview E: männlich, IT-Leiter, mittelständisches Unternehmen, angestellt, Biotechnologiebranche, Managementperspektive; 
$>$ Interview F: männlich, interner Berater, angestellt, Kleinunternehmen Dienstleistungsbranche, Projektperspektive;

> Interview G: weiblich, Führungskraft Finanzbereich, angestellt, mittelständisches Unternehmen Dienstleistungsbranche, Managementperspektive; und

> Interview $\mathrm{H}$ : weiblich, Projektmanagerin, angestellt, Behörde, Projektperspektive.

Die Interviews dauerten zwischen 26 und 61 Minuten.

Die Analyse der Interviews ist orientiert an der strukturierenden qualitativen Inhaltsanalyse (Mayring 2015, 65ff.) und erfolgte nach der Logik einer induktiven Kategorienbildung (ebd., 85ff.), auf die eine Interpretation anhand des Kategoriensystems angelehnt an die Forschungsfrage folgte.

\subsection{Ausgewählte empirische Ergebnisse}

Die Analyse des Datenmaterials erfolgte anhand der vorangehend theoretisch erarbeiteten Basis und enthält die Kategorien individuelle und organisationale Lernformen und ihre jeweiligen Bedingungen sowie pädagogische Technologien. Aufgrund der vorangehenden theoretischen Ausführungen hierzu wird hier auf eine wiederholende, einleitende Darstellung der Kategorien zugunsten der Vorstellung der Ergebnisse mit Einblicken in die Interviews verzichtet. Da organisationales Lernen auf der Basis individuellen Lernens erfolgt, werden zuerst einige Funde hieraus vorgestellt, bevor zu den pädagogischen Technologien und zum organisationalen Lernen übergeleitet wird.

\subsubsection{Ausgewählte empirische Einblicke zum individuellen Lernen}

Die Lernform, die unter anderem am meisten explizit als Lernen erwähnt wurde, ist das Erfahrungslernen der InterviewpartnerInnen. Der zweite Fall expliziten Erwähnens von Lernen ist das Erlernen des Scrum-Regelwerkes, wobei dies nicht zum Untersuchungsgegenstand zählt.

Das meiste Lernen im agilen Arbeitskontext von Scrum ist informell, wie im folgenden Interviewabschnitt deutlich wird:

»Ich würde sagen ja, also das Mindset, glaube ich, verändert sich auf jeden Fall bei Leuten der die Blickwinkeln, die Perspektiven erweitern sich vermutlich und dadurch erweitert, also das erweitert sich dann nicht nur für die eigene Arbeit, sondern auch für den Blick im Unternehmen « (JfP_Interview_F, Absatz 56).

Auch in Interview A wird das informelle Lernen thematisiert: 
»Das ist ein Lernprozess, des geht so mit Leuten, die aus einer klassischen Organisationsstruktur mit Abteilungsleitern und vielleicht noch Teamleiter dazwischen und so die dann halt ihre Aufgaben bekommen, diese abarbeiten müssen, ist das ein langer Lernprozess, aber grundsätzlich profitieren die erst mal davon, weil die sich auch selber entwickeln können und weil die ihr Wissen da einbringen «(JfP_Interview_A, Absatz 37).

Darüber hinaus kommt das transformative Lernen zur Sprache und verdeutlicht, dass sich Denk- und Verhaltensweisen innerhalb dieser Art zu arbeiten verändern können, was Lernprozesse impliziert:

»Insgesamt hat man deshalb nicht so richtig gespürt, weil es nur punktuell eingeführt wurde. Also in den zwei Jahren, in denen die oder fast zwei Jahren, in denen ich da war, wurde es nach Scrum punktuell eingeführt und vor allem, es gab unterschiedliche andere selbstorganisierte Projekte und überall da, wo das passiert ist, hat man gemerkt, dass die Menschen wieder viel mehr viel stärker Menschen werden. Und, das auch einer, der vorher sehr patriarchisch geführt hat, sich doch sehr vor allem für seine Verhältnisse emotional geöffnet hat und auch vorne sich hingestellt hat und Fehler eingestanden hat, also durchaus beispielhaft vorangegangen ist « (JfP_Interview_F, Absatz 28).

Hinweise für kooperatives Lernen können neben solchen für Um- und Dazulernen oder auch Erfahrungslernen ebenfalls entdeckt werden. Allerdings ist festzuhalten, dass in den Interviews genauso viele Belegbeispiele für individuelles Nicht-Lernen zu finden sind. In Interview $G$ wird dies explizit erwähnt: »Ich tue mir schwer zu sagen, dass jemand, wenn er Teil so eines Teams ist, sich danach auch verändert in seiner Arbeitsweise, ich glaube es nicht, nein «(JfP_Interview_G, Absatz 125).

Dabei ist es, wie auch in einem explizit pädagogischen Setting, bemerkenswert, dass die Rahmenbedingungen zum gelingenden Lernen beitragen oder es verhindern, wie in Interview D hervorgehoben wird: »außen herum Zwänge, Projektzwänge und innerhalb dieser Projektzwänge darfst du dann Scrum machen, und das funktioniert halt einfach nicht aus meiner Sicht « (JfP_Interview_D1, Absatz 27).

Bei den Rahmenbedingungen spielt die Organisation eine große Rolle, da sie den Kontext für Lernräume ermöglichen muss:

>Die einen wollen das, die sind total sind total ja fokussiert darauf, wirklich ihre ihren Part in dem Team einzunehmen. Und die anderen können nicht, weil sie ja durch äußere Beeinflussungen irgendwie abgehalten werden. Also die können nicht, weil sie vielleicht aus der Organisation heraus nicht das volle die volle Awareness geschaffen bekommen. Sie sind Scrum Master, ja, aber dann kannst du ja noch drei andere Projekte nebenher machen«(JfP_Interview_B, Absatz 26). 
Zu den in den Interviews auffindbaren Rahmenbedingungen zählen neben dem organisationalen Kontext auch TeilnehmerInnenorientierung (in dem Fall Teammitglieder), Kompetenzorientierung und Zielfokussierung.

\subsubsection{Ausgewählte empirische Einblicke zu pädagogischen Technologien}

Bemerkenswert ist, dass drei InterviewparterInnen sich gegen eine dogmatische Vorgehensweise hinsichtlich der Vorgaben des Programmes ausgesprochen haben und ein/e weitere/r InterviewpartnerIn flexibel je nach Zielerreichungsoption agiert. Die dazu führende Begründung war tatsächlich eine TeilnehmerInnen-, Ziel- und Kontextorientierung, die vor einer korrekten regelgeleiteten Anwendung eines Programmes stehe. Aber auch die Organisation, die eine agile Vorgehensweise tolerieren und zulassen muss, stellt einen Grund dar, nicht rein nach den Programmvorgaben zu arbeiten. Insofern kommt es in der Hälfte der Anwendungsfälle zu kombinierten Programmen, während die andere Hälfte regelgeleitet vorgeht und dies auch als Mehrwert sieht. Die einen fühlen sich begrenzt und konstatieren, das sei $\gg$ so engmaschig alles getaktet. Du hast überhaupt keine Freiheitsgrade mehr « (JfP_Interview_D2, Absatz 3). Die anderen bevorzugen einen Mix und sehen die Vorgaben nicht dogmatisch: $\gg$ Also ich find, also diese ganzen Sachen, ich finde es toll, weil man sich aus allem bedienen kann und sieht ich habe so ein großes Setting von Möglichkeiten. Es gibt viele Techniken, und so « (JfP_Interview_H, Absatz 52), während die anderen eher die Meinung vertreten, dass »[d] urch diesen regelmäßigen Austausch und durch diese feste Definition, dass man als Team zusammen ist, entwickelt sich in der Regel sehr schnell eine Dynamik « (JfP_Interview_G, Absatz 89), und dass ein striktes regelgeleitetes Vorgehen erst zum Erfolg führe.

Die meisterwähnten Veranstaltungsformen waren das Daily und die Sprints. Andere Scrum-Ereignisse kamen in den Interviews kaum zur Sprache und pädagogische Methoden wurden nicht erwähnt, Medien hingegen schon, wenn auch nur reduziert. So wurde doch über Burndowncharts, Kanbanboards oder Scrumboards berichtet. Die Rolle des Scrum Master wurde in Zusammenhang mit den Aktivitäten ebenfalls ausgeführt, wie hier in Interview B:

»Wichtig aber auch kann es sein, dass man wirklich einen guten, starken, erfahrenen Scrum Master auf ein junges Team loslässt oder auf also sagen wir mal ein unerfahrenes Team, vom Alter abgesehen, also ein junges unerfahrenes Team in der Thematik, dann braucht man einen starken Scrum Master, der auch viel noch Rollenverständnisse, Vorgehensweisen immer mal wieder auch erläutern kann « (JfP_Interview_B, Absatz 57).

Hier zeigt sich direkt die Verknüpfung der Technologien zu den pädagogischen Kernaktivitäten oder auch der Blick auf das Scrum-Team, das in der Lernerrolle gesehen 
wird. Der Scrum Master fungiert als MentorIn, als BegleiterIn oder unterrichtet, wie im oben genannten Beispiel oder auch nachfolgend in Interview $\mathrm{H}$ sichtbar wird:

$\gg$ Also ich vergleiche das gerne mit so einer Ameisenfarm. Dass ich denen die Handwerkszeuge gegeben und ein bisschen Input, also wie den wie den Zug auf den Gleis [...] oder auf die Schiene zu bringen und dann merke ich oh die fahren schon alleine los, prima. Und dann gucke ich. Und dann ist es vielleicht mal, sodass sich ein bisschen die Weiche nach links oder die Weiche nach rechts bringen, und das finde ich ganz prima « (JfP_Interview_H, Absatz 21).

Allerdings gilt hier auch, dass ein professioneller Umgang nötig ist, wie in Interview F festzustellen ist:

$\gg[\ldots .$.$] vielfach die Leute, nicht die richtige Begleitung dafür holen. Also so ein Scrum Mas-$ ter, der so ein bisschen Ahnung wirklich von einem Konzept hat, sondern einfach jemand. Ja, wir machen das jetzt mal danach, und Müller-Schulze kriegt dann noch den Auftrag, sich das noch mal durchzulesen und eine PowerPoint dazu zu erstellen, ist auch ein Punkt, warum das nicht funktioniert « (JfP_Interview_F, Absatz 42).

Die Betrachtung der pädagogischen Technologien zeigt, dass eine sehr bewusste Verwendung, oftmals sogar nach den pädagogischen Prinzipien der TeilnehmerInnensowie der Ziel- und Kontextorientierung, stattfindet und hierdurch eigentlich Lernräume möglichen sein sollten. Auch die Rollenbeschreibungen beinhalten pädagogische Kernaktivitäten, die in diesen Kontexten implizit pädagogisch genutzt werden und dennoch zu sichtlichen Veränderungen und individuellem Lernen führen:

»Was ich beobachte, ist, die blühen total auf. Also, wenn ich vergleiche die Menschen am Anfang im Projekt und am Ende, die sind ganz anders die sind viel offener, neugieriger, wacher. So wirkt das am Ende. Und vor allen Dingen, es ist also Irritation ist für mich als Mensch, der sich ja auch mit Psychologie viel beschäftigt hat, immer eine feine Sache, weil daraus entspringen ganz interessante vielfältige Möglichkeiten. Das ist denen nicht so klar, also für die ist Irritation und Neues, erst mal macht das Angst. Und nachher werden die auch mutiger, also ich finde das wunderbar« (JfP_Interview_H, Absatz 28).

\subsubsection{Ausgewählte empirische Einblicke zu organisationalem Lernen}

Vorweg festzuhalten ist, dass eher weniger empirische Belege für organisationales Lernen auffindbar sind. Wenn man mit der Brille von Argyris und Schön (1996) auf das Datenmaterial blickt, dann finden sich lediglich vereinzelt Hinweise auf single-looplearning, also für Lernen auf der organisationalen Handlungsebene, wie es zum Beispiel 
in Interview G deutlich wird: »dass diese Prioritäten sich zwar verschieben, aber dass Leute dennoch so starr in ihrer Denkensweise sind, dass man keinen richtigen Fortschritt erkennen kann «(JfP_Interview_G, Absatz 6).

Kollektive Realitätsdeutungen und -bewertungen, angelehnt an March und Olsen (vgl. Geißler 1995, 41ff.), sind in diesem Interview ebenfalls auffindbar:

»Wenn das Team Erfolg hat, dann kommt zum Schluss auch was Gutes raus. Und alle anderen Stakeholder, die irgendwie damit zu tun hatten, merken, dass es gut funktioniert hat. Und wenn es einmal passiert, ist super. Aber wenn es dann immer wieder passiert, dass man gut vorankommt, weil am Ende des Tages möchte man mit diesen Projekten ja auch irgendwas erreichen. Man hat Punkt A und dann irgendwann will man bei Punkt B sein. Und wenn man merkt, dass das funktioniert und greift, dann denke ich mal kommt auch der Lerneffekt, dass man merkt, das ist eine gute Art und Weise da vorzugehen « (JfP_Interview_G, Absatz 141).

Es kann also ein Lernprozess angestoßen werden, dessen Nach haltigkeit jedoch stark von der organisationalen Bewertung abhängt: »Aber ich glaube, dass durchaus es sein kann, dass Führungskräfte, die gute Erfahrung sehr gute Erfahrungen damit machen, mit anderen andere Führungskräfte vielleicht anstecken « (JfP_Interview_F, Absatz 52).

Allerdings muss konstatiert werden, dass das Datenmaterial wesentlich öfter Belege für organisationales Nicht-Lernen aufweist und die InterviewpartnerInnen dies auch deutlich artikulieren: »Es hat sich an der Organisation nichts geändert und und und dementsprechend auch an dem, an dem an dem Know-how nichts geändert « (JfP_Interview_D2, Absatz 15).

Diese Tatsache ist auf die organisationalen Bedingungen wie Führung, ökonomische Grundorientierung und erlernte Verhaltensweisen zurückzuführen. Die ersten zwei Bedingungen werden in Interview $\mathrm{H}$ angesprochen:

»Da sind die Geldgeber sehr wichtig und denen ist es egal, nach welcher Methode ein Unternehmen arbeitet. Denen war egal, ob die Mitarbeiter motiviert sind oder nicht. Und letztlich hat da eigentlich nur der der Output gezählt. Und wie viele wie viele Mitarbeiter kosten, wie viel Geld? Und was bringen die rein. Also den war die Methodik ganz egal. Da konnte man leider auch nicht dafür sorgen, dass eine Organisation lernt. Das hat immer an einer bestimmten Managementebene stopp gemacht « (JfP_Interview_H, Absatz 39-40)

\subsubsection{Fazit der empirischen Analyse}

Die empirische Analyse hat gezeigt, dass im vorhandenen Datenmaterial Bezüge zu individuellem Lernen zu finden sind. Allerdings weniger, als nach der theoretischen 
Analyse anzunehmen waren. Besonders das kooperative Lernen könnte in weiterführenden Studien fokussiert werden, da dies theoretisch einen wesentlichen Anteil der Handlungspraxis und des Neu-Lernens ausmachen sollte, in dem hier vorgestellten Material jedoch nicht stark vertreten war. Jedoch ist diese Erhebung wie vorab bereits angedeutet vielmehr der Versuch einer pädagogischen Feldexploration und gibt erste Hinweise außerhalb einer umfassenden Repräsentativität, sodass die hier vorgesellten Ergebnisse Anregungen für weitere Forschung geben sollen.

Das Programm Scrum eröffnet Lernräume, die in den Erfahrungsberichten der ExpertInnen überwiegend auf einer individuellen Ebene zu sehen sind und vermuten lassen, dass die Lernmöglichkeiten noch um einiges vielzähliger sind. Demzufolge könnte man erwarten, dass der Übertrag vom individuellen ins organisationale Lernen stattfindet. Dies kann jedoch nicht bestätigt werden. So deutet sich zwar vereinzelt organisationales Lernen an, jedoch sind die Hinweise für ein organisationales NichtLernen wesentlich deutlicher. Dies kann zurückgeführt werden auf die organisationalen Rahmenbedingungen, die zwar individuelle Entwicklung zulassen, jedoch keinen nachhaltigen Übertrag in kollektive Strukturen ermöglichen und/oder zulassen, unabhängig davon, ob Scrum in Reinform oder einer Hybridform angewendet wird. Es ist also zu resümieren, dass individuelles Lernen keine Garantie dafür ist, dass sich auch die Organisation dadurch weiterentwickelt.

Insofern ist es nicht verwunderlich, dass die ExpertInnen an vielen Stellen auch sehr deutlich über die Grenzen des Programms sprechen, die meist individuelle Voraussetzungen und Kompetenzen betreffen, aber auch die organisationale Rahmung:

»Was passt zu den Leuten, mit denen ich zusammenarbeite, sprich mit dem Team. Womit kann ich die leiten und lenken? Und was fällt denen eher schwer? Manchmal gibt es ja auch Teams, die wollen sich nicht verändern. Die haben eine strikte Vorgehensweise. Da kommt man agil sehr schnell an seine Grenzen, weil die damit nicht umgehen können « (JfP_Interview_H, Absatz 14).

Resümierend könnte es auch so auf den Punkt gebracht werden: Sowohl die Individuen als auch die Organisation bekommen durch den Einsatz von Scrum Lernmöglichkeiten geboten, müssen diese jedoch nicht wahrnehmen und nehmen diese auch noch nicht immer an. Insofern kann Pütz und Ricker zugestimmt werden, dass Agilität »durch eine Unternehmenskultur stimuliert [wird], die hierarchische Barrieren abbaut, Kontrolle durch Vertrauen ersetzt und autonomes sowie unternehmerisches Denken und Handeln fördert « $(2017,4)$, diese idealen Kontextbedingungen jedoch der Unternehmensrealität der ExpertInnen eher weniger entsprechen. Demzufolge beschreiben die acht interviewten Personen beispielsweise als Herausforderungen, dass 
$>$ Scrum in die vorhandene gesetzte organisationale Handlungspraxis integriert wurde und dort nicht entfaltet werden konnte (»Weil du einfach die die die Werkzeuge zwar in das Projektmanagement integriert hast, aber das eckt und beißt sich «; JfP_Interview_D1, Absatz 25-27);

$>$ trotz des Wunsches nach agilem Arbeiten die Rahmenbedingungen nicht verändert wurden ( $\gg$ Aber die die Management-Voraussetzungen, die organisatorischen Voraussetzungen haben sich nicht geändert. Und dadurch sind die auch gescheitert «; JfP_Interview_A, Absatz 54);

$>$ Scrum als Allheilmittel angepriesen, jedoch nach kurzer Zeit auch die neue ScrumBesetzung den organisationalen Voraussetzungen angeglichen wurde (» habe ich auch sehr gehofft damals, als die kamen, weil die hatten ja hohes Management Standing und so weiter die Jungs und ich dachte na ja, wenn die jetzt sagen, sie brauchen zehn Leute Fulltime für zwei Scrum-Teams, dann kriegen die die, haben die auch gedacht. Ich hab' das ein Jahr probiert oder länger und bin nicht zum Erfolg gekommen und dann am Ende des Tages auch nicht, also es ist wieder so ein Konstrukt rausgekommen, wie ich eben die ganze Zeit hatte «; JfP_Interview_A, Absatz 54).

Diese Herausforderungen zeigen, dass die Organisationskultur sowie die vorhandenen Handlungspraktiken und Gegebenheiten starken Einfluss auf das gelingende agile Arbeiten nehmen und dies nicht einfach per Programmatik eingeführt auch in einen Erfolg mündet. Ergo, eine agile Organisation ist nicht immer gleichzeitig auch eine lernende Organisation, zumindest nicht aus einer erziehungswissenschaftlichen Betrachtung. Hierzu ist jedoch auch anzumerken, dass es bei der Anwendung von Scrum im organisationalen Kontext nicht um Lernen per se geht, sondern dies mit agilem Arbeiten im Praxisdiskurs zu Unrecht verknüpft zu sein scheint.

\section{Resümee}

Agilität ist mittlerweile allgegenwärtig in der Unternehmenspraxis, in Managementdiskursen und sozialen Netzwerken. Oft wird es als Allheilmittel für eingefahrene Teamkonstellationen oder aus dem Ruder laufende Projekte angepriesen. Führungskräfte fordern die Umstellung auf agiles Arbeiten meistens, ohne Kontext und Passung zu analysieren. Eine fundierte wissenschaftliche Auseinandersetzung aus einer lerntheoretischen Perspektive ist bislang nicht zu finden. Der vorliegende Artikel hatte sich deshalb zum Ziel gesetzt, der Frage nachzugehen, wie und wo das Lernen hin $z u$ der neuen Verhaltensweise erfolgt und welche Lernformen und pädagogischen Technologien hierfür im Alltag der AnwenderInnen zum Tragen kommen, wenn innerhalb 
einer Organisation agil nach Scrum gearbeitet wird. Für das alltägliche Arbeiten sollte hinterfragt werden, inwieweit die Scrum zugrunde liegenden Arbeitsweisen eine erziehungswissenschaftliche Nähe aufweisen. So sollte ein erster explorativer Blick gewagt werden.

Hierfür erfolgte zuerst eine theoretische Analyse des Scrum-Rahmenwerkes, auf dessen Basis acht ExpertInneninterviews geführt und analysiert wurden. Es ist somit abschließend möglich, die Frage zu beantworten, welche Lernprozesse aus erziehungswissenschaftlicher Sicht stattgefunden haben und welche Formen organisationalen Lernens und damit organisationaler Weiterentwicklung vonstattengingen. Insofern können sowohl Grenzen, Herausforderungen als auch Möglichkeiten agilen Projektmanagements retrospektiv erziehungswissenschaftlich bewertet sowie eine Aussage darüber getroffen werden, inwieweit Scrum nachhaltiges organisationales Lernen ermöglicht.

Da das Programm verschiedene Lernformen, pädagogische Aktivitäten und Technologien zu enthalten scheint und somit Lernräume bietet, könnte die Hypothese zugrunde gelegt werden, dass organisationales Lernen, wie zum Beispiel die Verankerung von Agilität, unter anderem durch kollektives oder kooperatives Lernen möglich wäre.

Es kann jedoch abschließend festgehalten werden, dass die zahlreichen Lernräume, die Scrum sowohl auf individueller und damit auch organisationaler Ebene eröffnet, von den Akteuren noch nicht ausgenutzt werden. Das heißt, dass die reine theoretische Anwesenheit von Lernräumen keine Garantie dafür ist, dass Lernen stattfindet. Deshalb ist zu empfehlen, nicht allein aufgrund der theoretischen und praktischen Anwesenheit von individuellem Lernen, zum Beispiel in kooperativen Lernformen, davon auszugehen, dass auch die Organisation (nachhaltig) lernt. Eine agil arbeitende Organisation muss nicht unbedingt eine lernende Organisation sein. Agiles Arbeiten kann losgelöst von organisationaler Weiterentwicklung vonstattengehen. Im vorliegenden Datenmaterial konnten zum Beispiel lediglich single-loop-Lernschleifen nachgewiesen werden. Die theoretisch möglichen deutero-Lernprozesse auf Reflexionsebene waren nicht ersichtlich. Deshalb ist anzunehmen, dass die Verwendung von Scrum keinen nachhaltigen Effekt auf die gesamtorganisationale Entwicklung nimmt, da unter Umständen das vorhandene Potenzial nicht genutzt wird. Grund hierfür könnte sein, dass die Umsetzung des Programmes technokratisch und nicht kontextsensibel erfolgt. Auch die strikte Anwendung eines Programmes muss nicht dazu führen, dass Lernen stattfindet. Pütz und Ricker haben hierzu analysiert, in welchen Ausprägungen Mitarbeiter zu Veränderungen bereit sind $(2017,5)$. Hinsichtlich der Erfolgsfaktoren von Scrum (Böhm 2019) kann es hilfreich sein, sich weiterführend mit gelingendem Lernen in agilem Arbeiten auseinanderzusetzen, um hierüber die organisationale Entwicklung zu beeinflussen und an Erfolgs- und Lernfaktoren zu koppeln. Auch die Verknüpfung von 
Zufriedenheit (Komus 2013) und Lernen in nachfolgenden Studien kann aufschlussreich für eine nachhaltige Umsetzung von Agilität sein.

Es kann also abschließend festgehalten werden, dass der schnelle Wandel, in dem Organisationen sich heute zumeist befinden, Agilität erfordert, diese jedoch auf Mitarbeiterebene nicht nachhaltig anzukommen scheint und wahrscheinlich über agile Arbeitsformen auch nicht zwingend herstellbar ist. Insofern ist die Unterscheidung zwischen Agilität und agilem Arbeiten wahrscheinlich größer, als angenommen. Organisationslernen muss aus keinem der beiden erfolgen. Fraglich bleibt, ob ein Wandel ohne nachhaltiges individuelles und organisationales Lernen langfristig ausreicht, um im Markt zu überleben.

\section{Literatur}

Argyris, Chris und Donald A. Schön. 1996. Die Lernende Organisation. Grundlagen, Methoden, Praxis. Stuttgart: Klett-Cotta.

Beck, Kent, Mike Beedle, Arie van Bennekum, Alistair Cockburn, Ward Cunningham und Martin Fowler 2001. Manifest für agile Softwareentwicklung. Zugriff 29.09.2020. https://agilemanifesto. org/iso/de/manifesto.html.

Böhm, Janko. 2019. Erfolgsfaktor Agilität. Warum Scrum und Kanban zu zufriedenen Mitarbeitern und erfolgreichen Kunden führen. Wiesbaden: Springer Vieweg.

Deitering, Franz G. 1995. Selbstgesteuertes Lernen. Göttingen: Verlag für Angewandte Psychologie.

Dinkelaker, Jörg. 2018. Lernen Erwachsener. Stuttgart: Kohlhammer.

Dohmen, Günther. 2001. Das informelle Lernen. Die internationale Erschließung einer bisher vernachlässigten Grundform menschlichen Lernens für das lebenslange Lernen aller. Bonn: Bundesministerium für Bildung und Forschung.

Duméril, Jean-Christophe. 2019. »Agility Suitability Check. Agile Methoden am richtigen Ort einsetzen«. In Führen in der Arbeitswelt 4.0, hrsg. v. Christoph Negri, 51-61. Berlin: Springer.

Förster, Kerstin und Roy Wendler. 2012. »Theorien und Konzepte zu Agilität in Organisationen«. Dresdner Beiträge zur Wirtschaftsinformatik Nr. 63. Zugriff 29.09.2020. https://tud.qucosa.de/ api/qucosa\%3A27335/attachment/ATT-0/.

Frey, Tatjana. 2012. „Veranstaltungsformen«. In Klinkhardt Lexikon Erziehungswissenschaft, Bd. 3, hrsg. v. Klaus-Peter Horn, Heidemarie Kemnitz, Winfried Marotzki und Uwe Sandfuchs, 356. Bad Heilbrunn: Klinkhardt.

Friedrichs, Henrike. 2012. »Medien«. In Klinkhardt Lexikon Erziehungswissenschaft, Bd. 2, hrsg. v. Klaus-Peter Horn, Heidemarie Kemnitz, Winfried Marotzki und Uwe Sandfuchs., 347-49. Bad Heilbrunn: Klinkhardt.

Geißler, Harald. 1995. Grundlagen des Organisationslernens. 2. durchges. Aufl. Weinheim: Deutscher Studien-Verlag.

Geißler, Harald. 2000. Organisationspädagogik. Umrisse einer neuen Herausforderung. München: Vahlen.

Giesecke, Herman. 2015. Pädagogik als Beruf. Grundformen pädagogischen Handelns. 12. überarb. Aufl. Weinheim: Beltz Juventa.

Göhlich, Michael. 2014a. »Aus Erfahrung lernen«. In Pädagogische Theorien des Lernens, 2. Aufl., hrsg. v. Michael Göhlich, Christoph Wulf und Jörg Zirfas, 191-202. Weinheim: Beltz Juventa. 
Pädagogische Elemente in agilen Methoden - inwieweit lernen Organisationen im agilen Arbeiten?

Göhlich, Michael. 2014b. »Organisationales Lernen«. In Pädagogische Theorien des Lernens, 2. Aufl. hrsg. v. Michael Göhlich, Christoph Wulf und Jörg Zirfas, 222-32. Weinheim: Beltz Juventa.

Göhlich, Michael, Christoph Wulf und Jörg Zirfas. 2014. Pädagogische Theorien des Lernens. 2. Aufl. Weinheim: Beltz Juventa.

Hörmann, Georg. 1996. »Selbstbewertungsprozesse«. In Handbuch Selbstorganisiertes Lernen, hrsg. v. Siegfried Greif und Hans-Jürgen Kurtz, 149-54. Göttingen: Verlag für Angewandte Psychologie.

Kade, Jochen, Dieter Nittel und Wolfgang Seitter. 1999. Einführung in die Erwachsenenbildung/Weiterbildung. Stuttgart u. a.: Kohlhammer.

Kipper, Jenny. 2014. Die lernende Organisation. Personalentwicklung und Qualitätsmanagement pädagogische Wissensformen und Technologien in Großunternehmen. Berlin: epubli.

Koch, Lutz. 1999. »Lernen«. In Pädagogik-Lexikon, hrsg. v. Gerd Reinhold, Guido Pollak und Helmut Heim, 351-55. München: Oldenbourg Verlag.

Konrad, Klaus und Silke Traub. 2010. Kooperatives Lernen. Theorie und Praxis in Schule, Hochschule und Erwachsenenbildung. Baltmannsweiler: Schneider Verlag Hohengehren GmbH.

Koschek, Holger. 2014. Geschichten von Scrum. Von Sprints, Retrospektiven und agilen Werten. 2. überarb. Aufl. Heidelberg: dpunkt Verlag.

Koschek, Holger. 2018. Neue Geschichten von Scrum. Von Führung, Lernen und Selbstorganisation in fortschrittlichen Unternehmen. Heidelberg: dpunkt Verlag.

Komus, Ayelt. 2013. »Agile Methoden in der Praxis - Studie zur Anwendung und Zufriedenheit«. HMD - Praxis der Wirtschaftsinformatik 50 (290): 84-91.

Komus, Ayelt et al. 2020. Studie Status Quo (Scaled) Agile 2019/20. Koblenz. Zugriff 28.09.2020. https:// www.hs-koblenz.de/index.php?id=7169.

Krancher, Oliver, Pascal Luther und Marc Jost. 2018. »Key Affordances of Platform-as-a-Service: Self-Organization and Continuous Feedback«. Journal of Management Information Systems 35 (3): 776-812. Zugriff 15.01.2021. https://www.researchgate.net/publication/324115137_ Key_Affordances_of_Platform-as-a-Service_Self-Organization_and_Continuous_Feedback.

Majkovic, Anna-Lena, Ellen Gundrum, S. M. Benz, N. Dzsula und Rafael Huber. 2019. IAPStudie 2019. Agile Arbeits- und Organisationsformen in der Schweiz. Ergebnisse der qualitativen Interviews. Zürich: IAP Institut für Angewandte Psychologie der ZHAW Zürcher Hochschule für Angewandte Wissenschaften. Zugriff 29.09.2020. https://www.zhaw.ch/storage/psychologie/ upload/iap/studie/IAP-Studie_Agilitaet_2019.pdf.

Maximini, Dominik. 2018. Scrum - Einführung in der Unternehmenspraxis. Von starren Strukturen zu agilen Kulturen. 2. Aufl. Berlin: Springer Gabler Verlag.

Mayring, Philipp. 2015. Qualitative Inhaltsanalyse. Grundlagen und Techniken. 12. Aufl. Weinheim \& Basel: Beltz Verlag.

Meuser, Michael und Ulrika Nagel. 1994. »Expertenwissen und Experteninterview«. In Expertenwissen. Die institutionalisierte Kompetenz zur Konstruktion von Wirklichkeit, hrsg. v. Ronald Hitzler, Anne Honer und Christoph Maeder, 180-92. Opladen: Westdeutscher Verlag.

Negri, Christoph. 2019. Führen in der Arbeitswelt 4.0. Berlin: Springer.

Nittel, Dieter. 1996. „Die Pädagogisierung der Privatwirtschaft und die Ökonomisierung der öffentlich verantworteten Erwachsenenbildung«. Zeitschrift für Pädagogik 42 (5): 731-50. Weinheim: Beltz Verlag.

Nittel, Dieter, Nikolaus Meyer und Jenny Kipper. 2020. »Ordnungsdimensionen pädagogischer Situationen«. Zeitschrift für Pädagogik 66 (3): 382-400. Weinheim: Beltz Verlag.

Peters, Christoph, Benedikt Simmert, Karen Eilers und Jan Marco Leimeister. 2019. Future Organisation Report 2019. St. Gallen. Zugriff 25.09.2020. https://www.campana-schott.com/de/de/ unternehmen/media-events/studien/future-organization-report/2019. 
Peters, Christoph, Benedikt Simmert, Karen Eilers und Jan Marco Leimeister. 2020. Future Organisation Report 2020. St. Gallen. Zugriff 25.09.2020. https://www.campana-schott.com/de/de/ unternehmen/media-events/studien/future-organization-report.

Pfister, Andres und Prisca Müller. 2019. »Psychologische Grundlagen des agilen Arbeitens«. In Führen in der Arbeitswelt 4.0, hrsg. v. Christoph Negri, 33-50. Berlin: Springer.

Pütz, Horst und Saskia Ricker. 2017. Kulturwandel in der Digitalen Transformation messen und gestalten. Zugriff 29.09.2020. https://www.greatplacetowork.de/events-and-great-blog/blog/ agilitaet-in-deutschen-unternehmen-es-gibt-noch-luft-nach-oben/.

Reinhold, Gerd, Guido Pollak und Helmut Heim. 1999. Pädagogik-Lexikon. München \& Wien: Oldenbourg.

Rump, Jutta und Klaus Breitschopf. 2018. HR-Report 2018. Schwerpunkt Agile Organisationen auf dem Prüfstand. Weinheim: ABT Print und Medien. Zugriff 29.09.2020. https://www.hays.de/ hr-report-2018.

Schwaber, Ken und Jeff Sutherland. 2017. The Scrum Guide. Deutsche Version. Zugriff 29.09.2020. https://www.scrumguides.org/download.html.

Schwarzer, Christine und Petra Buchwald. 2014. »Umlernen und Dazulernen«. In Pädagogische Theorien des Lernens, 2. Aufl., hrsg. v. Michael Göhlich, Christoph Wulf und Jörg Zirfas, 191-202. Weinheim: Beltz Juventa.

Siebert, Horst. 2010. »Lernen«. In Wörterbuch Erwachsenenbildung, 2. überarb. Aufl., hrsg. v. Rolf Arnold, Sigrid Nolda und Ekkehard Nuissl, 190-92. Bad Heilbrunn: UTB.

Siebert, Horst. 2012. Didaktisches Handeln in der Erwachsenenbildung. Didaktik aus konstruktivistischer Sicht. 7. aktualis. u. erw. Aufl. München: Wolters Kluwer.

Traub, Silke und Klaus Konrad. 2016. Kooperatives Lernen: Theorie und Praxis in Schule, Hochschule und Erwachsenenbildung. 6. unveränd. Aufl. Baltmannsweiler: Schneider Verlag.

Vidgen, Richard und Xiaofeng Wang. 2009. „Coevolving Systems and the Organization of Agile Software Development«. Information Systems Research 20(3): 355-76. Zugriff 15.01.2021. http:// hdl.handle.net/10379/1861.

Zeuner, Christine. 2012. »Transformative Learning«: Ein lerntheoretisches Konzept in der Diskussion«. In Erwachsenenbildung und Lernen, hrsg. v. Heide von Felden, Christiane Hof und Sabine Schmidt-Lauff, 93-104. Baltmannsweiler: Schneider Verlag.

\section{Die Autorin}

Jenny Kipper, Dr. phil, ist Diplompädagogin mit Fokus auf Erwachsenenbildung. Sie lehrt als Dozentin (Hochdeputat) an der Goethe-Universität Frankfurt und ist als Coach und Organisationsberaterin tätig. Ihre Arbeitsschwerpunkte sind organisationales Lernen und Organisationstheorien, Change Management, Persönlichkeitsentwicklung und qualitative Forschungsmethoden.

Kontakt: Dr. Jenny Kipper, Institut für Sozialpädagogik und Erwachsenenbildung, Fachbereich Erziehungswissenschaften, Goethe-Universität, Theodor-W.-Adorno-Platz 6, 60323 Frankfurt am Main; E-Mail: kipper@em.uni-frankfurt.de 